\title{
Transport through graphene nanoribbons: suppression of transverse quantization by symmetry breaking
}

\author{
Florian Libisch * Stefan Rotter, and Joachim Burgdörfer \\ Institute for Theoretical Physics, Vienna University of Technology \\ Wiedner Hauptstraße 8-10/136, A-1040 Vienna, Austria, European Union
}

\begin{abstract}
We investigate transport through nanoribbons in the presence of disorder scattering. We show that size quantization patterns are only present when $S U(2)$ pseudospin symmetry is preserved. Symmetry breaking disorder renders transverse quantization invisible, which may provide an explanation for the necessity of suspending graphene nanoconstrictions to obtain size quantization signatures in very recent experiments. Employing a quasi-classical Monte-Carlo simulation, we are able to reproduce and explain key qualitative features of the full quantum-mechanical calculations.
\end{abstract}

PACS numbers: 73.23.-b, 73.63.-b, 73.40.-c

Graphene, the first two-dimensional solid [1], features remarkable electrical and mechanical properties that open the possibility for many new and intriguing applications [2] including high precision mechanical or chemical sensors, ultrafast single-electron transistors, and spintronic devices. Graphene quantum dots can meanwhile be fabricated with well-defined dimensions [3, 4] allowing for the observation of Coulomb blockade [5, 6, and Klein tunneling-related phenomena [7.

While properties of the perfect honeycomb lattice and its consequences for electronic structure and dynamics are well understood, the effect of disorder caused by local distortions of the lattice symmetry, graphene-substrate interaction, or charged impurities on the unique properties of graphene remains the focus of theoretical investigations 8 11. On general grounds, local symmetry breaking in low-dimensional systems is expected to have more pronounced effects than in 3D bulk materials. In this Letter, we demonstrate the remarkably strong sensitivity of quantum transport through graphene nanoribbons to very low concentrations of point defects that break the $\mathrm{SU}(2)$ pseudospin symmetry associated with the triangular sublattices of graphene. We simulate transport through disordered nanoribbons of realistic size described by a tight binding Hamiltonian. To interpret our numerical results we use two simpler models: a continuous Dirac-like equation and a quasi-classical Monte-

(a) Single vacancy
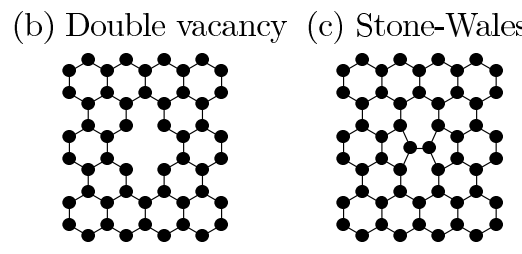

FIG. 1: Defects in graphene nanoribbons (a) single vacancy breaking the $A B$-sublattice symmetry, (b) double vacancy preserving the sublattice symmetry, (c) Stone-Wales deformation: four hexagons are replaced by two pentagons and two heptagons which also breaks $A B$-sublattice symmetry.
Carlo simulation. We identify pseudospin non-conserving scattering at lattice vacancies to be the key for the breakdown of size quantization.

The ideal, infinitely extended graphene sheet features a honeycomb lattice made up of two interleaved triangular sublattices (A and B). It can be described in tightbinding approximation by the Hamiltonian 12 .

$$
H=\sum_{i, s}\left|\phi_{i, s}\right\rangle V_{i}\left\langle\phi_{i, s}\left|-\sum_{(i, j), s} \gamma_{i, j}\right| \phi_{i, s}\right\rangle\left\langle\phi_{j, s}\right|+h . c .
$$

where the sum $(i, j)$ extends over pairs of lattice sites, $\left|\phi_{j, s}\right\rangle$ is the tight-binding orbital with spin $s$ at lattice site $j, V_{i}$ is a locally varying potential, and $\gamma_{i, j}$ is the hopping matrix element between lattice sites $i$ and $j$. For improved accuracy, we describe the hexagonal graphene lattice using third-nearest-neighbor coupling (for details see Ref. 13). We are interested in the effects of bulk scattering at localized point defects and consider samples of ribbons with an average width of up to $60 \mathrm{~nm}$ corresponding to $\approx 300$ unit cells in transverse direction $(y)$ orthogonal to the direction of transport $(x)$. We use an approach suitable for the efficient description of large-scale graphene nanodevices employing a variant of the modular recursive green's function method (MRGM) 13, 14. Different defects (see Fig. 1) can be easily included at module boundaries. We perform ensemble averages over, typically, 100 different disorder realizations to eliminate any non-generic features.

We analyze the implications of the numerical results with the help of simpler models. One of them employs the Dirac-like bandstructure of graphene. Close to the Fermi energy, the band structure of Eq. (1) can be approximated (assuming that $V_{i} \ll \gamma_{i, j}$ ) by a conical dispersion relation around the $K$ point [15],

$$
E\left(k+k_{K}\right)=E\left(k_{K}\right)+k \partial_{k} E\left(k_{K}\right)+\mathcal{O}\left(k_{K}^{2}\right) \approx v_{\mathrm{F}}|k|,
$$

with the Dirac-like Hamiltonian,

$$
H=\hbar v_{\mathrm{F}}\left(\begin{array}{cc}
0 & \partial_{x}+i \partial_{y} \\
\partial_{x}-i \partial_{y} & 0
\end{array}\right)+\mathbb{1} \cdot V(\mathbf{x})
$$


where we have set $E\left(k_{K}\right)=0$. Equation (3) ignores both the length scale of the graphene lattice constant $a=1.4$ $\AA$ and the broken rotational symmetry of the cone due to the hexagonal lattice structure, an effect known as triangular warping [2, 16. Eigenfunctions of Eq. (3) on an infinitely extended sheet $(V=0)$ are plane waves $|k\rangle$ where the direction of motion $\theta_{k}$,

$$
\theta_{k}=\tan ^{-1}\left(k_{y} / k_{x}\right),
$$

is coupled to the $A B$-sublattice degree of freedom [2,

$$
|\mathbf{k}\rangle=e^{i \mathbf{k} \cdot \mathbf{r}}\left(|A\rangle+e^{i \theta_{k}}|B\rangle\right) / \sqrt{2} .
$$

The Hamiltonian $H$ preserves the $\mathrm{SU}(2)$ pseudospin projection (or helicity) $h=(\boldsymbol{\sigma} \cdot \mathbf{k}) /|k|$ along $\hat{k}$, i.e. the angle $\theta_{k}$ ( $\boldsymbol{\sigma}$ is the vector of the Pauli matrices). Furthermore, the band structure features two non-equivalent cones ("valleys") at the $K$ and $K^{\prime}$ points in the reciprocal lattice. This additional degeneracy allows to represent the low-energy band-structure near $E=0$ in terms of Dirac-like four-spinors $|\psi\rangle=\left(\psi_{A}^{K}, \psi_{B}^{K}, \psi_{A}^{K^{\prime}}, \psi_{B}^{K^{\prime}}\right)$ with amplitudes for the $A B$-sublattice in real space and for the $K K^{\prime}$ points in reciprocal space. The sign of $\theta_{k}$ is reversed upon transition from $K$ to $K^{\prime}$. Note that physical spin is not included in the present analysis.

One of the consequences of the preservation of pseudospin is the suppression of backscattering [2]. If the scattering potential commutes with the helicity operator, the first-order transition probability $P$ for scattering $|\mathbf{k}\rangle \rightarrow\left|\mathbf{k}^{\prime}\right\rangle$ is proportional to

$$
P\left(\mathbf{k} \rightarrow \mathbf{k}^{\prime}\right)=\left|\left\langle\mathbf{k}^{\prime}|V| \mathbf{k}\right\rangle\right|^{2} \propto \cos ^{2}\left[\left(\theta_{\mathbf{k}}-\theta_{\mathbf{k}^{\prime}}^{\prime}\right) / 2\right],
$$

which vanishes for $\left|\theta_{\mathbf{k}}-\theta_{\mathbf{k}^{\prime}}^{\prime}\right|=\pi$, i.e. for backscattering. For locally broken $A B$-sublattice symmetry [Eq. (5)], the pseudospin is no longer conserved and backscattering becomes possible. Consequently, differential cross sections for scattering at local defects that preserve the $\mathrm{SU}(2)$ pseudospin symmetry should obey Eq. (6) while defects that locally destroy the $A B$-sublattice allow for scattering in arbitrary direction, in particular for isotropic $s$ wave scattering in the long-wavelength limit $(k \rightarrow 0)$,

$$
P\left(\mathbf{k} \rightarrow \mathbf{k}^{\prime}\right) \propto \text { const. }
$$

To elucidate the consequences of these specific scattering features we incorporate them in a quasi-classical transport simulation based on the propagation of Monte-Carlo ensembles of classical trajectories: pseudospin conserving lattice defects are simulated by elastic scattering probabilities of the form of Eq. (6), pseudospin non-conserving defects will be represented by isotropic $s$-wave scattering [Eq. (7)]. We randomly shoot trajectories that move classically (i.e. on straight lines) in between scattering events. After traversing a mean free path $\lambda_{s}$ (determined by the disorder concentration), a scattering event with either pseudospin conserving or non-conserving angular differential scattering probability takes place. Note that the only quantum input are the differential scattering probabilities Eq. (6) and Eq. (7). A trajectory is counted as transmitted (reflected) if it traverses the length $L \gg \lambda$ (returns past the starting point).

As initial condition we choose the longitudinal wave numbers $k_{x, n}$ corresponding to the quantized open modes $n$, for each energy $E$

$$
k_{x, n}=\sqrt{\left(\frac{E}{\hbar v_{F}}\right)^{2}-\left(\frac{n \pi}{W}\right)^{2}}, \quad n \in \mathbb{Z} .
$$

We use, typically, 200.000 trajectories per open channel with initial momenta chosen according to Eq. (8). If the mismatch $\left|k_{y}-k_{y, n}\right|$ between the transverse wave number $k_{y}$ and $k_{y, n}$ corresponding to the largest flux-carrying mode (i.e. the largest $n$ for which $k_{x, n}$ is real) is larger than $\left|k_{y}-k_{y, n+1}\right|$, scattering into an evanescent mode (i.e. complex $k_{x}$ ) is assumed, initiating a new scattering event in backwards direction $\left(-k_{x}, k_{y}\right)$. The average over ensembles of trajectories provides a quasi-classical Monte-Carlo estimate for the conductance as a function of energy.

We consider three different lattice defects (Fig. 1) which locally perturb the electronic structure and, thus, introduce disorder. The simplest defect is a point defect residing on a single carbon atom. Such a defect can be caused, for example, by chemical absorption of hydrogen, forming a covalent bond with the $p_{z}$ orbital of a carbon atom, locally changing the electronic configuration from $s p^{2}$ to $s p^{3}$. Consequently, the $p_{z}$ orbital of this carbon atom no longer contributes to the electronic bandstructure of graphene. In a tight-binding approximation, this can be modeled by a single (electronic) lattice vacancy, i.e. one carbon atom is effectively removed from the graphene lattice [see Fig. 1(a)]. We consider an ensemble average over many configurations of randomly placed point defects with a relative defect density as small as $n_{i}=10^{-5}$ impurities/carbon. As a second class of defects we consider double vacancies, i.e. we remove both atoms of a unit cell [see Fig. 1(b)]. In contrast to a single vacancy, this defect does not break the $A B$-sublattice symmetry. Double vacancies are pseudospin conserving as both the $\mathrm{A}$ and the $\mathrm{B}$ lattice are equally affected by them. A third and more complex defect of the ideal graphene lattice is the Stone-Wales deformation (SWd): Four hexagons are replaced by two heptagon-pentagon pairs [see Fig. 1.(c)]. As a consequence, the $A B$-sublattice symmetry is broken, and $A B$-scattering occurs. To first order approximation, we adapt the tight-binding parameters of the graphene ribbon to model the coupling parameters at the SWd using geometry-dependent coupling parameters.

We perform quantum transport simulations for a zigzag graphene nanoribbon of width $W=60 \mathrm{~nm}$ and 


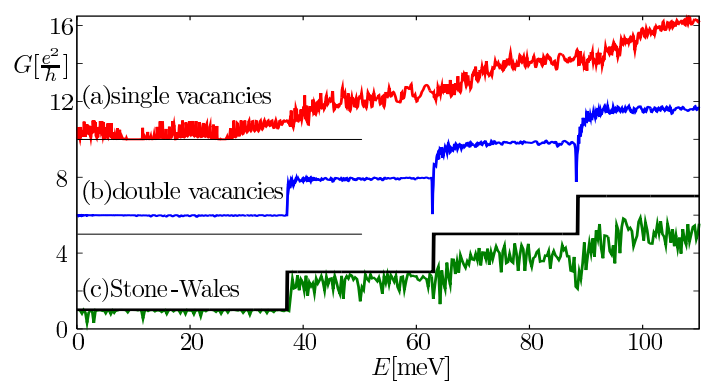

FIG. 2: (Color online) Conductance of a $60 \mathrm{~nm}$ wide graphene nanoribbon with a length of $1 \mu \mathrm{m}$ and a defect density $n_{i}=$ $10^{-5}$ defects/atom of (a) single-point vacancies, (b) double vacancies, or (c) Stone-Wales deformations (curves vertically offset by $5 e^{2} / h$ for clarity). The staircase function for ideal size quantization plateaus is shown in (c) as thin black line.

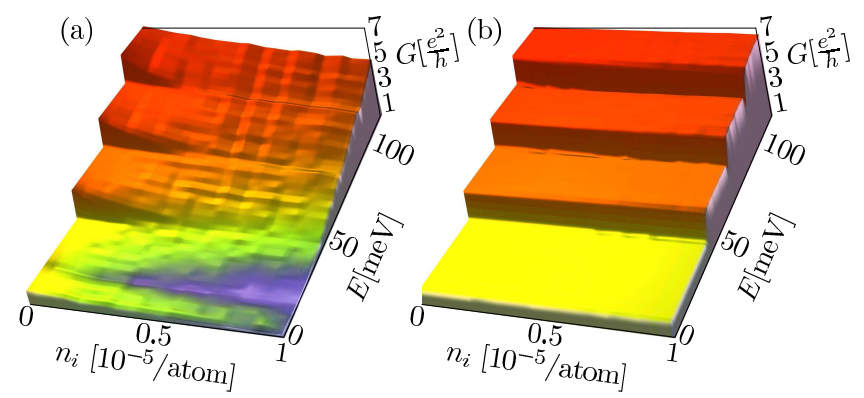

FIG. 3: (color online) Conductance through a graphene zigzag nanoribbon of length $1 \mu \mathrm{m}$ and width $W=60 \mathrm{~nm}$ as a function of energy and disorder concentration $n_{i}$ (in units of $10^{-5}$ defects/atom) averaged over 100 disorder realizations for (a) single vacancies and (b) double vacancies.

length $L=1 \mu \mathrm{m}$ in the presence of disorder. "Bulk" disorder is introduced by randomly distributed electronic lattice defects. Even relative defect concentrations as low as $n_{i}=10^{-5}$ defects/atom [Fig. 2 give rise to pronounced deviations from the ideal staircase-shaped conductance $G$ with plateaus due to transverse size quantization. While both single- and double vacancies lead to a reduction of transmission, the two types of vacancies give rise to very different modifications. While pseudospin conserving double vacancies approximately preserve the feature of quantization plateaus, with reduced height and pronounced dips near the steps where additional modes open, the quantization plateaus are completely washed out for single vacancies. This is all the more remarkable as the total number of point defects for single vacancies is only half the number for the double vacancies. This clearly points to the breaking of the $A B$ sublattice symmetry as origin of the loss of size quantization. The drastic difference between pseudospin conserving double vacancies and non-conserving single vacancies persists over a wide range of defect concentrations and is robust against an average over many disorder configurations (Fig. 3).
The connection between pseudospin conservation and transverse quantization steps can be inferred from the relation between the direction of the wave vector [Eqs. $45[5]$ and the helicity operator acting on the $\mathrm{SU}(2)$ representation-space spanned up by the $\mathrm{A}$ and $\mathrm{B}$ sublattices. For the free Dirac equation [Eq. (3)] the ratio between $k_{x}$ and $k_{y}$ is determined by the pseudospin [see Eq. (5)]. In turn, transverse quantization for a finitewidth ribbon relates the step quantum number $n$ with $k_{y}$. Through the introduction of defects that break pseudospin conservation, or interactions with an underlying substrate, which invariably introduces a spatially varying electronic environment $V(\mathbf{x})$ [see Eq. (3)] breaking $A B$-symmetry, the transverse quantum number $n$ becomes ill defined, resulting in the strong suppression of transverse quantization steps. Conversely, the absence of pronounced size-quantization plateaus in experiment [17 20] hints at broken $A B$-symmetry in experimental structures. This mechanism can be illustrated and verified with the help of our quasi-classical trajectory simulations. Using either an angular scattering probability at point defects that are pseudospin preserving [Eq. [6] ] or non-conserving [Eq. (7)], we can reproduce all qualitative features of quantum conductance in remarkable detail (Fig. 4): for $s$-wave scattering, quantization steps are strongly suppressed, giving an (approximately) linear slope of transmission with energy. For pseudospinconserving scattering, quantization steps are pronounced, and the characteristic dips in the transmission near the thresholds observed in the full quantum mechanical calculation are reproduced. These dips resulting from scattering into evanescent modes disappear in the case of $s$ wave scattering, as the backscattered trajectory "forgets" its new direction after the next scattering event. We have verified that these striking differences between single- and double vacancy scattering are not qualitatively changed by higher-order effects (e.g. triangular warping). The relation between broken $A B$-sublattice symmetry and the destruction of size-quantization plateaus implies that for disorder caused by different and more complex defects which also result in pseudospin non-conserving scattering, signatures of transverse quantization should also disappear. This can, indeed, be verified for Stone-Wales defects [Fig. 11(c)]. The size quantization plateaus are washed out by as few as $1 \mathrm{SWd}$ in $10^{5}$ atoms [see Fig. 2(c)].

The role of pseudospin conservation in the persistence of transverse quantization can also be extracted from the scattering wave functions which we obtain from the full quantum calculation. In order to identify structures in the scattering wave function as a function of ribbon length, we average over the $y$ component of the scattering wave function of a single incoming mode $n$

$$
\left\langle\left|\psi_{n}(x)\right|^{2}\right\rangle=\frac{1}{W} \int_{0}^{W}\left|\psi_{n}(x, y)\right|^{2} \mathrm{~d} y
$$




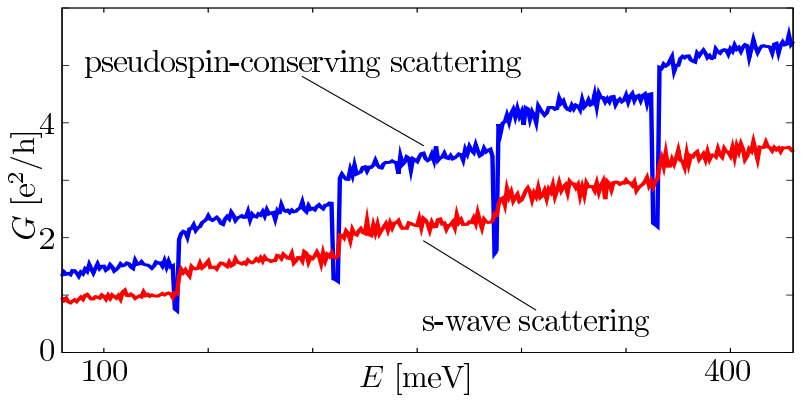

FIG. 4: (Color online) Quasi-classical Monte-Carlo simulation of transport through a disordered nanowire, using a Dirac-like linear dispersion relation, and either graphene pseudospinconserving scattering (upper blue line) or $s$-wave scattering (lower red line) at randomly distributed local defects.

For disorder created by single-vacancy defects [Fig. [5(a)], the transmitted wave function in the exit lead does not feature pronounced oscillations since it represents a superposition of many transverse modes. By contrast, the scattering states for a disordered nanoribbon with pseudospin conserving double vacancies feature in this region well discernible oscillating patterns on two length scales [see Fig. [5(b)]: (i) the short beating period of $\lambda=0.7$ $\mathrm{nm}$, corresponding to the distance (in $k$ space) between the $\Gamma$ and $K$ point (see Ref. [21]) and (ii) a much slower variation with a length scale $\Lambda \approx 12 \mathrm{~nm}$ [see Fig. [5(b)] corresponding to the wavelength associated with the linear dispersion relation $E=v_{\mathrm{F}} \hbar k$ (i.e., the distance from the $K$ point to a given point on the Dirac cone). These two length scales differ by almost two orders of magnitude. The persistence of these two well-defined oscillatory components supports the notion that the presence of disorder does not destroy size quantization provided disorder scattering is pseudospin conserving.

To summarize, we have presented full quantum transport simulations through disordered graphene nanoribbons of realistic size, and find that even very low concentrations of defects that destroy the $A B$-sublattice symmetry lead to destruction of transverse size quantization plateaus. By contrast, randomly distributed defects that preserve the $A B$-sublattice symmetry leave the quantization plateaus intact while modifying the transmission function. Our present results suggest that the difficulty in observing pronounced quantization plateaus in the conductance can be motivated by to the presence of pseudospin non-conserving defects. This finding suggests that the variation in local electronic structure of graphene due to interactions with an underlying substrate or with local chemisorbates binding to individual carbon atoms could be sufficient to destroy size quantization plateaus in the experiment $17-20$. Indeed, in agreement with these results, very recent studies 22] demonstate that plateaus of quantized conductance can be observed if graphene quantum point contacts are both suspended from the substrate as well as thoroughly annealed to reduce the number of adsorbates on the graphene lattice.
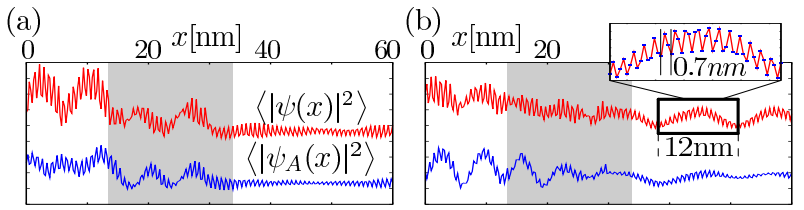

FIG. 5: (Color online) Averaged wave function density $\left\langle\left|\psi_{1}(x)\right|^{2}\right\rangle$ [see Eq. [9]] and sublattice density $\left\langle\left|\psi_{1, A}(x)\right|^{2}\right\rangle$ of sub-lattice $A$ for the scattering through a $10 \mathrm{~nm}$ wide disordered graphene nanoribbon (disordered area shaded) featuring ten (a) single vacancy [(b) double vacancy] defects.

We thank K. Ensslin, J. Güttinger, and C. Stampfer for valuable discussions. Support by the Austrian Science Foundation (Grant No. FWF-P17359) and ViCoM SFB041 is gratefully acknowledged. Numerical calculations were performed on the Vienna scientific cluster (VSC).

* Electronic address: florian.libisch@tuwien.ac.at

[1] K. S. Novoselov et al, Nature 438, 197 (2005).

[2] A. H. C. Neto et al., Rev. Mod. Phys. 81, 109 (2009).

[3] L. A. Ponomarenko et al., Science 320, 356 (2008).

[4] J. Güttinger et al., Appl. Phys. Lett. p. 212102 (2008).

[5] C. Stampfer, E. Schurtenberger, F. Molitor, J. Güttinger, T. Ihn, and K. Ensslin, Nano Lett. 8, 2378 (2008).

[6] J. Güttinger et al., Phys. Rev. Lett. 103, 046810 (2009).

[7] M. I. Katsnelson, K. S. Novoselov, and A. K. Geim, Nature Physics 2, 620 (2006).

[8] M. Wimmer, A. R. Akhmerov, and F. Guinea, Phys. Rev. B 82, 045409 (2010).

[9] E. R. Mucciolo, A. H. CastroNeto, and C. H. Lewenkopf, Phys. Rev. B 79, 075407 (2009).

[10] S. Ihnatsenka and G. Kirczenow, Phys. Rev. B 80, 201407R (2009).

[11] F. Libisch, S. Rotter, J. Güttinger, C. Stampfer, and J. Burgdörfer, Phys. Rev. B 81, 245411 (2010).

[12] P. R. Wallace, Phys. Rev. 71, 622 (1947).

[13] F. Libisch, S. Rotter, and J. Burgdörfer (2010), to be published.

[14] S. Rotter, J. Z. Tang, L. Wirtz, J. Trost, and J. Burgdörfer, Phys. Rev. B 62, 1950 (2000).

[15] G. W. Semenoff, Phys. Rev. Lett. 53, 2449 (1984).

[16] E. McCann et al., Phys. Rev. Lett 97, 146805 (2006).

[17] M. Y. Han, J. C. Brant, and P. Kim, Phys. Rev. Lett. 104, 056801 (2010).

[18] F. Molitor, A. Jacobsen, C. Stampfer, J. Güttinger, T. Ihn, and K. Ensslin, Phys. Rev. B 79, 075426 (2009).

[19] Y.-M. Lin, V. Perebeinos, Z. Chen, and P. Avouris, Phys. Rev. B 78, 161409 (2008).

[20] E. R. Mucciolo and C. H. Lewenkopf, J. Phys. Cond. Matt 22, 273201 (2010).

[21] F. Libisch, C. Stampfer, and J. Burgdörfer, Phys. Rev. B 79, 115423 (2009).

[22] N. Tombros et al., (2011), arXiv:1102.0434. 